\title{
El Capital Social y la Integración de Procesos en la Gestión de las Cadenas de Abastecimiento en el Sector Real en Colombia
}

\author{
Juan C. Aldana-Bernal ${ }^{(1)}$ y César A. Bernal-Torres ${ }^{(2) *}$ \\ (1) Centrum Católica Graduate Business School, Jr. Daniel Alomía Robles n 125 Urbanización Los Álamos de \\ Monterrico, Surco, Lima, Perú (e-mail: jcaldana@pucp.pe). \\ (2) Universidad de La Sabana, Escuela Internacional de Ciencias Económicas y Administrativas, Km. 7, Autopista Norte \\ de Bogotá, Puente del Común, Campus Universitario, Chía, Colombia (e-mail: cesar.bernal@unisabana.edu.co).
}

${ }^{*}$ Autor a quien debe ser dirigida la correspondencia

Recibido Dic. 12, 2018; Aceptado Feb. 14, 2019; Versión final Mar. 21, 2019, Publicado Oct. 2019

\section{Resumen}

Esta investigación evalúa la relación del capital social con la integración de procesos en la gestión de cadenas de abastecimiento en empresas del sector real, en Colombia, mediadas por el tamaño organizacional y la incertidumbre del entorno. Para esto se encuestaron 232 empresas asociadas a dicho sector y, con la información obtenida, se realizó un análisis multivariante de ecuaciones estructurales con las herramientas SPSS y AMOS. Como resultado de ello se evidencia una relación directa significativa del capital social sobre la integración de procesos en la gestión de cadenas de abastecimiento, lo cual permite identificar al capital social como un determinante fundamental en los esfuerzos de integración de la cadena de abastecimiento, no así de las variables mediadoras (tamaño e incertidumbre del entorno).

Palabras clave: cadenas de abastecimiento; integración de procesos; capital social; capital estructural; capital cognitivo; capital relacional.

\section{Social Capital and the Integration of Processes in the Supply Chain Management in the Real Sector in Colombia}

\begin{abstract}
This research evaluates the relationship of social capital with the integration of processes in the supply chain management in companies of the real sector in Colombia, mediated by their organizational size and the uncertainty of the environment. For this purpose, 232 companies associated to the real sector in Colombia were surveyed and with the information obtained, multivariate analysis of structural equations was made with the SPSS and AMOS tools. As a result, there is a significant direct relationship between social capital and the integration of processes in the supply chain management, thus social capital was identified as a fundamental determinant in efforts to integrate the supply chain, but not the mediating variables analyzed (size and uncertainty of the environment)
\end{abstract}

Keywords: supply chain; process integration; social capital; structural capital; cognitive capital; relational capital 


\section{INTRODUCCIÓN}

La integración es un proceso de coordinación y control de las partes de un sistema mediante flujos y vínculos de largo plazo que se establecen entre una organización y una o más organizaciones en su entorno. En el contexto de las organizaciones empresariales, una de las formas de integración más importante se ha identificado en la gestión de las cadenas de abastecimiento (SCM por sus siglas en inglés), entendida como la integración de los procesos fundamentales del negocio de las empresas, relacionados con el suministro de productos y la prestación de servicios desde los proveedores primarios hasta el cliente final (Flynn et al., 2010). Así, un alto nivel en la integración interempresarial ha demostrado ser uno de los factores con alta incidencia en el desempeño y la sostenibilidad organizacional (Naslund y Hulthen, 2012). La integración de procesos en la gestión de la cadena de abastecimiento ( $\mathrm{SCl}$ por sus siglas en inglés) es un factor importante para el desarrollo empresarial, por esto se hace necesario evaluar los determinantes que llevan a las organizaciones a iniciar este proceso. En este sentido, Lambert y Cooper (2000) han identificado dos tipos de factores como determinantes de la integración de estas cadenas: a) los físicos y técnicos, caracterizados por ser tangibles y fácilmente medibles, y b) los comportamentales, caracterizados por ser intangibles y difíciles de medir. Sin embargo, aunque existen diversos estudios que analizan los factores físicos y técnicos (o duros) que permiten la integración en la SCM, no es así con los factores comportamentales o blandos, y en particular con el Capital Social (CS) en estos procesos.

Adicionalmente, de acuerdo con Katunzi (2011), la mayoría de las barreras de la SCl corresponden a factores blandos, entre estos el capital social (CS), que se ha asociado positivamente con las relaciones de la SCM (Roden y Lawson, 2014; Son et al., 2016), pero que no ha sido suficientemente estudiado como determinante en la SCl (Bastas et al., 2018; Borgatti y Li, 2009). En este sentido, se han identificado variables que pueden mediar en el proceso de la SCI. Por un lado, el tamaño (S) de la empresa respecto a las otras con las que establece un proceso de integración, parece ser una variable asociada a la integración interorganizacional. Por otro lado, la incertidumbre del entorno (UE) en donde se desenvuelven las empresas puede facilitar o restringir que la SCI se desarrolle adecuadamente (Giménez et al., 2012). De acuerdo con lo anterior, esta investigación analizó, la relación entre el capital social (CS) y la integración de procesos en la gestión de cadenas de abastecimiento (SCl) en el sector real colombiano, moderada por el tamaño de las empresas (S) y la incertidumbre del entorno (UE), con el propósito de generar conocimiento sobre la realidad de la SCl en países emergentes como Colombia, donde poco se ha estudiado el tema. La información para el análisis de las hipótesis se obtuvo de una encuesta, medida en escala Likert, aplicada a una muestra de 232 empresas de diversos tamaños y sectores económicos. Los datos fueron procesados mediante análisis multivariante de ecuaciones estructurales (SEM) con las herramientas SPSS y AMOS. Los resultados evidencian una fuerte relación entre el CS y la SCI, pero no se evidencia la mediación del tamaño (S) ni de la incertidumbre del entorno (UE) en esa relación.

\section{OTROS ANTECEDENTES}

La gestión de la cadena de abastecimiento (SCM) se ha definido como una forma de integrar la oferta y la demanda mediante la planeación, gestión y control de todos los eslabones de la cadena para proporcionar valor añadido al cliente final (Lockamy y McCormack, 2004). La integración ha sido identificada como una de las características esenciales en la SCM dado que usualmente se describe como la interacción a través de interfaces normalizadas con el fin de reducir el esfuerzo requerido para el procesamiento de la información, entre los socios de la cadena (Mentzer et al., 2001), mediante la asociación de empresas de un mismo sector para generar eficiencias que impactan al cliente final.

\section{Integración en la gestión de la cadena de abastecimiento}

En general, se han encontrado dos grandes dimensiones para catalogar los procesos de integración interempresarial. Por un lado, la integración de procesos internos, que corresponde a la conectividad y simplificación de las funciones propias de la empresa, cuyo propósito es eliminar la estructura burocrática 0 por silos y, así, mejorar la coordinación entre áreas funcionales para satisfacer la demanda del mercado (Morash y Clinton, 1998). Por otro lado, la integración de procesos externos hace referencia a la conectividad y simplificación en la forma de desarrollar actividades conjuntas con proveedores, distribuidores y clientes, y la integración interna se asume como un prerrequisito para la integración externa en la SCM (Horn et al, 2014). Sin embargo, a pesar de que hay diversas propuestas para catalogar la integración interorganizacional, una de las propuestas que ha tenido mayor aceptación, es la planteada por Flynn et al. (2010). Estos autores sugieren que la $\mathrm{SCl}$ es un nivel en el cual las empresas colaboran estratégicamente con sus socios de negocios, a través de procesos inter e intra organizacionales, con el propósito de alcanzar la eficiencia y eficacia en los flujos de productos, servicios, dinero, información y decisiones, procurando generar el máximo valor al cliente. Su propuesta presenta la SCI bajo tres dimensiones: (a) integración interna, (b) integración con proveedores o integración hacia atrás, y (c) integración con clientes o integración hacia adelante. 
Por otra parte, los estudios sobre la integración de los procesos de la cadena de abastecimiento (SCl) han evidenciado diferentes barreras que la afectan (Katunzi, 2011) tales como: (a) la mentalidad de silo al no considerar el impacto de sus acciones sobre los otros; (b) la falta de visibilidad en la cadena de suministro al no trabajar de manera coordinada; (c) la falta de confianza pues no se crea una buena imagen y reputación entre los miembros de la cadena; y (d) la falta de conocimiento dado que las empresas no comparten ni colaboran con sus pares en la cadena. Se identifica que estas barreras corresponden a factores blandos e impiden a las empresas alcanzar mejores resultados empresariales, por lo que su estudio se hace necesario. En este contexto, es esencial entender la SCM como una red social (Naslund y Hulthen, 2012) en donde un grupo de organizaciones independientes se relacionan en forma estrecha (en los dos sentidos: vertical y horizontal), colaborando consciente y cooperativamente con cada otra en aspectos técnicos y comportamentales, mediante un intercambio repetitivo y en el largo plazo, para gestionar el conocimiento (Inkpen, et al., 2005). Esto permite un mejor desempeño y el desarrollo de ventajas competitivas que no podrían ser alcanzadas individualmente por las empresas (Borgatti y Li, 2009).

\section{El capital social y la integración}

El capital social (CS) ha sido asociado al desarrollo de las redes sociales y, por tanto, es muy pertinente para esta investigación (Nahapiet y Ghoshal, 1998). El CS considera el conjunto de recursos actuales y potenciales que posee una persona o una organización, como un resultado o consecuencia de su pertenencia a una red de relaciones (Inkpen et al., 2005). Así, el CS propone que los vínculos establecidos en las redes permiten acceder a recursos y, por ende, el principal beneficio para el desarrollo de una organización es la oportunidad de adquirir conocimiento de otros miembros de la red (Adler y Kwon, 2002). La gestión de este conocimiento facilita la interacción social a los miembros de la red y es un factor clave para el desarrollo de las funciones organizacionales y de la SCI en los procesos de planeación y control (Simanca et al., 2015). Por esto, se reconoce en la teoría del CS un alto potencial para el análisis de la SCM (Autry y Griffis, 2008). En este sentido, el CS ha sido definido bajo tres dimensiones: (a) estructural, (b) cognitiva, y (c) relacional (Nahapiet y Ghoshal, 1998). La dimensión del capital estructural (SC) hace referencia a los patrones de relacionamiento entre los actores de una red y evalúa cómo los miembros de la red crean o no vínculos con otros actores, en otras palabras, esta dimensión mide la configuración de la red empresarial para generar oportunidades de transacción en la red. La dimensión de capital cognitivo (CC) representa aspectos que dan una identidad compartida a los miembros de la red, definidos por las metas, la cultura y el lenguaje compartido entre las partes (Inkpen et al., 2005). La dimensión de capital relacional (RC) hace referencia a los elementos que permiten establecer y mantener los vínculos entre los miembros de la red asociados con el comportamiento tal como el respeto y la amistad. Las facetas claves de esta dimensión son la confianza, para la perpetuación de las relaciones exitosas, la integridad y el compromiso (AlSagheer et al., 2011).

Diversos estudios han encontrado una relación positiva entre el CS y las interacciones entre proveedores y compradores en la SCM (Son et al., 2016; Villena et al., 2011), mientras que otros estudios han identificado una relación positiva entre el CS y la mejora en la innovación y el costo de los compradores en la SCM, y resultados positivos en el desempeño del negocio (Roden y Lawson, 2014). También el estudio de Horn et al. (2014) analiza las dimensiones del CS y la integración empresarial desde una perspectiva interna y externa, para determinar su efecto en el éxito del abastecimiento global. Como resultado, identificaron una relación positiva entre las dimensiones del CS interno con la integración empresarial interna, así como en las dimensiones del CS externo con la integración empresarial con proveedores. Sin embargo, son pocos los estudios encontrados en la literatura que relacionen el CS y la SCM según lo evidencia la revisión realizada por Matthews y Marzec (2011) sobre la aplicación del concepto de CS en estudios de operaciones. De otra parte, el tamaño de una organización es un factor que permite a la organización tener mayor reconocimiento en el mercado y administrar más amplios recursos. Las organizaciones grandes pueden estar más propensas a tener una interacción más compleja en las SCM, lo que les ocasiona mayor vulnerabilidad ante la incertidumbre y, por lo tanto, tendrían mayores dificultades para integrarse dado que la incertidumbre en el entorno (UE) de una organización puede cambiar sustancialmente los procesos de SCl (Giménez et al., 2012). Boon-itt y Wong (2010) proponen cuatro dimensiones de la incertidumbre del entorno: (a) incertidumbre del proveedor, (b) incertidumbre del cliente, (c) incertidumbre de la competencia, e, (d) incertidumbre de la tecnología, y encuentran que existe una relación proporcional pero inversa entre el ambiente de complejidad y el nivel de SCI. Por lo tanto, este estudio identifica el tamaño y la incertidumbre del entorno como variables moderadoras porque podrían constituirse en factores que facilitan o dificultan la relación entre el CS y la SCI.

En Latinoamérica, y especialmente en Colombia, son muy pocos los estudios relacionados con el CS, los cuales han encontrado fortalezas en las relaciones sociales de pequeñas empresas, pero debilidades en el perfil relacional asociado al trabajo en equipo y a la cooperación interna y externa. En lo referente a SCI, Ruiz, Mahmoodi y Ayala (2011) encontraron que la colaboración y la integración son un factor crítico de éxito para las cadenas de abastecimiento en Latinoamérica, por lo cual sugieren la necesidad de la integración de las pymes en Colombia para incrementar su competitividad. De otra parte, la investigación de Roldán (2015) 
sobre las problemáticas de las cadenas de abastecimiento en Latinoamérica encontró que es necesario desarrollar políticas y estrategias unificadas para cada sector empresarial con el fin de internacionalizarse. De la misma manera, según el estudio de Torres y García (2008) acerca de la gobernanza en las SCM en Colombia, uno de los principales impedimentos encontrados para establecer alianzas estratégicas en la SCM es la falta de confianza entre las partes.

\section{Hipótesis}

Con base en los planteamientos de Nahapiet y Ghoshal (1998) el CS sería un factor determinante en los recursos relacionales entre las empresas en una SCM; los estudios de Roden y Lawson (2014), Son et al. (2016) y Villena et al. (2011) afirman que existe relación positiva entre el CS y la colaboración en la SCM, especialmente entre proveedores y compradores; Horn et al. (2014) relacionan el CS y la integración desde perspectivas interna y externa y, Aldana-Bernal et al. (2018) proponen una relación directa entre los factores blandos, entre ellos el CS y la SCI. Para este estudio se propuso la relación entre CS y SCl que se ilustra en la figura 1.

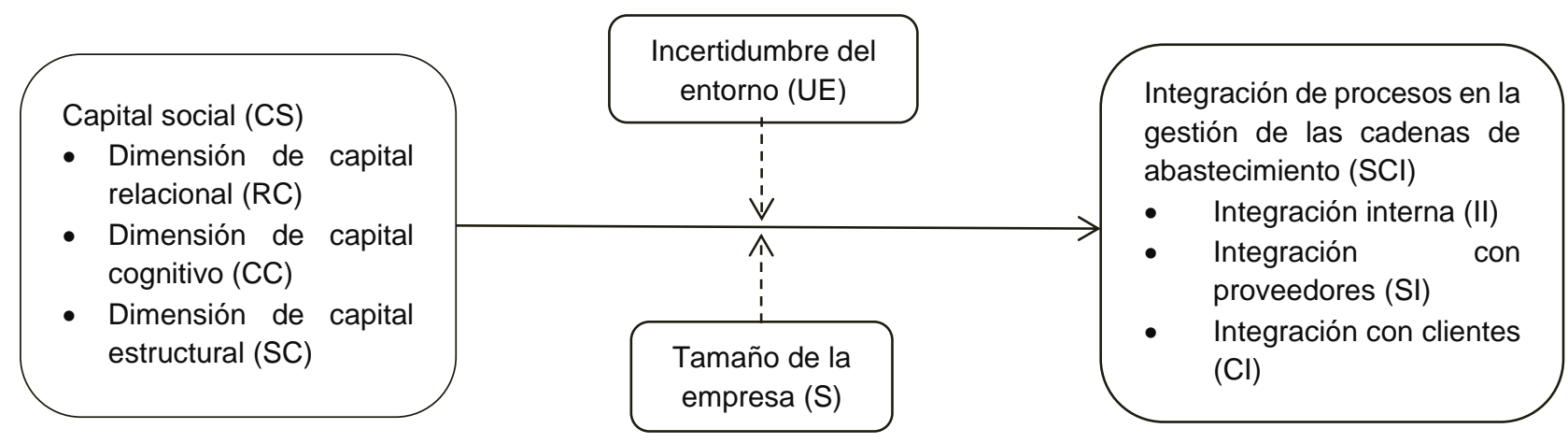

Fig. 1: Relación entre capital social con la integración de los procesos de la gestión de las cadenas de abastecimiento con mediación del tamaño de las empresas y la incertidumbre del entorno.

De acuerdo con los hallazgos de los estudios mencionados, se plantean las siguientes hipótesis: H1: El CS está relacionado positiva y significativamente con la $\mathrm{SCl}$ en las empresas asociadas al sector real en Colombia; H2: El RC está relacionado positiva y significativamente con la SCl en las empresas asociadas al sector real en Colombia; H3: El CC está relacionado positiva y significativamente con la SCI de las empresas asociadas al sector real Colombia; H4: El SC está relacionado positiva y significativamente con la SCl de las empresas asociadas al sector real en Colombia; H5: El S de las organizaciones afecta de forma inversamente proporcional la relación entre el CS y la SCI de las empresas asociadas al sector real en Colombia; y H6: La UE afecta de forma inversamente proporcional la relación entre el CS y la SCI de las empresas asociadas al sector real en Colombia.

\section{METODOLOGÍA}

Las empresas participantes en el estudio fueron seleccionadas por muestreo por conveniencia, a partir de contactos personales de los investigadores y a través del envío de correos electrónicos personalizados a ejecutivos de empresas. Junto con la encuesta, se envió un documento a cada empresa invitada, que contenía el propósito del estudio, algunos lineamientos para el diligenciamiento, información de los investigadores, y la garantía de confidencialidad en el manejo de la información proporcionada.

\section{Población}

Luego de reiterados contactos para recordar la invitación a diligenciar la encuesta, de un total 350 empresas invitadas a participar del estudio, se obtuvo el diligenciamiento de 261 cuestionarios, a los cuales, posteriormente se verificó la calidad de los datos, y se validaron 232 encuestas para su respectivo procesamiento. Esta muestra representa empresas de diversos sectores destacándose el de productos alimenticios $(11,6 \%)$, químico y farmacéutico $(7,8 \%)$, automotriz $(6,5 \%)$, metalmecánico $(4,7 \%)$, confecciones y calzado $(4,7 \%)$. De igual forma, en lo referente al tamaño de las empresas la muestra presenta una importante representatividad, conteniendo el $44,4 \%$ de grandes empresas, $25,0 \%$ de microempresas, $15,4 \%$ de medianas empresas y $15,1 \%$ de pequeñas empresas. Con respecto al rol de las empresas, se encontró una participación de un $45,7 \%$ para fabricantes, $29,7 \%$ para los proveedores y 24,6 para los distribuidores y comercializadores dentro de la muestra. 


\section{Instrumento}

El cuestionario utilizado para recolectar la información fue construido a partir de cuestionarios de estudios previos consultados en la revisión de la literatura antes mencionada, los cuales habían validado las variables y las escalas. La validación de la traducción y entendimiento del cuestionario se realizó a través de una discusión abierta con un grupo de profesionales (estudiantes de posgrado) y mediante consultas a un grupo de expertos, docentes-investigadores. Posteriormente, se aplicó una prueba piloto del cuestionario a un grupo de estudiantes de posgrado. Los cuestionarios (en escala Likert de 1 a 7 ) fueron diligenciados de manera presencial (157) y de forma virtual (104) para un total de 261 cuestionarios. Para garantizar la calidad de los datos, se utilizaron los procedimientos de verificación de la diversidad de los respondientes y revisión de casos repetidos. Por un lado se eliminaron 18 cuestionarios que correspondían a empresas que no están asociadas a la cadena de abastecimiento del sector real colombiano. Por otro lado, dado que la unidad de análisis es la empresa, otras nueve encuestas se eliminaron por casos repetidos en los cuales más de un ejecutivo de la misma empresa respondió el cuestionario. Finalmente, otras encuestas fueron desechadas por la ausencia de datos de interés (Hair et al., 2004), utilizando para los análisis 232 encuestas. El proceso de recolección de información se realizó desde abril de 2017 a marzo de 2018.

\section{Variables de medición}

La variable dependiente para esta investigación corresponde al nivel de integración de procesos de gestión de la cadena de abastecimiento (SCI). Según Flynn et al. (2010), la integración en la SCM se mide de acuerdo con el grado de colaboración estratégica de una empresa con sus aliados de la cadena, mediante la gestión efectiva de sus procesos internos y externos, de tal manera que garantice eficientemente los flujos de información, productos, servicios, dinero y decisiones, maximizando el valor proporcionado al cliente final y minimizando el costo y tiempo requeridos. Para este análisis se consideraron las tres dimensiones propuestas por estos autores: (a) integración interna, (b) integración con proveedores, y (c) integración con clientes. La integración interna se entiende como el grado en el cual una empresa estructura sus propias estrategias, prácticas y procesos mediante un sistema colaborativo y sincronizado para cumplir completamente los requerimientos del cliente (Flynn et al., 2010). Esta dimensión se evaluó a través de nueve indicadores. La integración con proveedores y con clientes se entiende como el grado en el cual una empresa estructura con sus proveedores externos y con sus clientes estratégicos, respectivamente, prácticas y procesos mediante sistemas colaborativos y sincronizados e involucra el desarrollo de competencias fundamentales con proveedores y clientes críticos. La integración con proveedores se evaluó mediante doce indicadores y la integración con clientes mediante once indicadores. Para la operacionalización de estas variables se emplearon los cuestionarios de Flynn et al. (2010).

La variable independiente, capital social (SC), se define como un conjunto de recursos actuales y potenciales que posee una organización como consecuencia y derivado de su pertenencia a una red de relaciones (Nahapiet y Ghoshal, 1998; Inkpen et al., 2005). Para la operacionalización de esta variable se emplearon los enunciados de los cuestionarios desarrollados por Son et al. (2016) y Villena et al. (2011) que han sido validados y probados. Esta variable, según Nahapiet y Ghoshal (1998), se desarrolla a través de tres factores: (a) capital relacional, (b) capital cognitivo, y (c) capital estructural. El capital relacional (RC), que hace referencia al compromiso y confianza en el relacionamiento, se evaluó a través de cinco indicadores. El capital cognitivo (CC) está asociado con una visión compartida entre los miembros de un sistema social y fue evaluado mediante cuatro indicadores. Finalmente, el capital estructural (SC), asociado a la configuración de contactos, se midió a través de cinco indicadores. Con respecto a la variable moderadora, correspondiente al tamaño (S) de las organizaciones, la operacionalización se realizó con base en la clasificación de las empresas en Colombia según las leyes 590 de 2000 y 905 de 2005 (Mincit, 2017), de acuerdo a las cuales una microempresa tiene entre 1 y 10 personas, una pequeña empresa entre 11 y 50 personas, una mediana entre 51 y 200 personas, y una gran empresa cuenta con más de 200 trabajadores directos. De otra parte, la variable incertidumbre del entorno se evaluó según la aproximación que realiza Boon-itt y Wong (2010) como un factor de contingencia que afecta la SCl. Así se adoptó la operacionalización que sugiere cuatro dimensiones para la incertidumbre ambiental: (a) incertidumbre de los proveedores, con cuatro indicadores; (b) incertidumbre de los clientes, con dos indicadores; (c) incertidumbre de los competidores, con dos indicadores; y (d) incertidumbre tecnológica, con tres indicadores. Esta herramienta ha sido probada y validada en el entorno de la SCI.

\section{RESULTADOS}

El procesamiento de los datos recolectados se realizó con el software IBM SPSS Statistics V21 para realizar el análisis de fiabilidad (Alfa de Chronbach) y de unidimensionalidad de los factores presentados, al igual que para el Análisis Exploratorio (EFA, por sus siglas en inglés) y Confirmatorio de Factores (CFA, por sus siglas en inglés). Para los modelos de CFA y ecuaciones estructurales se empleó la extensión AMOS del SPSS 
V25. El modelo de ecuaciones estructurales (SEM, por sus siglas en inglés) integra un conjunto de técnicas multivariantes que examina simultáneamente un conjunto de relaciones de dependencia (Hair et al., 2004). Se emplea este modelo porque cuenta con dos beneficios principales. Primero tiene la capacidad de medir variables observables y no observables directamente, y sus errores. Segundo permite evaluar las relaciones desde el análisis exploratorio al confirmatorio y contrastar desde un modelo a gran escala hasta una teoría (Hair et al., 2004). En la tabla 1 se presenta la información empleada para los análisis desarrollados en este estudio.

Tabla 1: Información empleada para los análisis

\begin{tabular}{|c|c|c|c|c|}
\hline Concepto & $\begin{array}{l}\text { No. } \\
\text { Encuestas }\end{array}$ & $\begin{array}{l}\text { No. Variables } \\
\text { latentes } 2^{\circ} \\
\text { Orden }\end{array}$ & $\begin{array}{l}\text { No. Variables } \\
\text { latentes 1er. } \\
\text { Orden }\end{array}$ & Indicadores \\
\hline Diligenciadas & 261 & & & 57 \\
\hline Depuradas & 232 & & & 57 \\
\hline Análisis de Unidimensionalidad & 232 & & 10 & 57 \\
\hline Análisis de Fiabilidad & 232 & & 10 & 57 \\
\hline Análisis Factorial Exploratorio (EFA) & 60 & & & 57 \\
\hline Análisis Factorial Confirmatorio (CFA) & 172 & 2 & 6 & 32 \\
\hline CFA - CS & 172 & 1 & 3 & 19 \\
\hline $\mathrm{CFA}-\mathrm{SCl}$ & 172 & 1 & 3 & 13 \\
\hline \multicolumn{5}{|l|}{ Modelos de Ecuaciones Estructurales } \\
\hline Modelo 1 - Impacto del RC en la SCI & 232 & 1 & 4 & 17 \\
\hline Modelo 2 - Impacto del CC en la SCI & 232 & 1 & 4 & 16 \\
\hline Modelo 3 - Impacto del SC en la SCI & 232 & 1 & 4 & 18 \\
\hline Modelo 3 - Impacto del SC en la SCl & 232 & 2 & 6 & 25 \\
\hline
\end{tabular}

\section{Análisis de Fiabilidad y Unidimensionalidad de Factores}

El análisis de unidimensionalidad se realizó para determinar si cada uno de los indicadores converge hacia el factor evaluado y cuenta con un ajuste aceptable, para esto se empleó como criterio de adecuación de la muestra el Kaiser Meyer Olkin (KMO) que muestra si las correlaciones entre índices son suficientemente pequeñas, recomendándose que estén por encima de 0,8, y desechando valores por debajo de 0,5 (Byrne, 2010). La significancia debe ser cercana a 0 y el método utilizado, por ser el mejor evaluado, es el de máxima verosimilitud (Hoyle, 2015). El análisis de fiabilidad se realizó por cada una de las variables latentes primarias (no medibles directamente) del modelo teórico y muestra que las dimensiones del CS convergen hacia los factores con índices KMO mayores de 0,777. En lo referente a las dimensiones del SCl, la SI se observa bastante adecuada, pero los indicadores de las variables II y $\mathrm{Cl}$ cargan hacia dos factores cada una, aunque con indicadores KMO altos (superiores a 0,873 ). Todos los factores se identifican como significativos. Para las dimensiones de la incertidumbre, dos de los factores (incertidumbre del cliente y competidores), no tienen indicadores suficientes y están en el nivel de desecharlos. Los otros dos factores presentan correlaciones significativas y KMO por debajo de 0,789.

Posteriormente se realizó el análisis de fiabilidad interna con el Alfa de Cronbach para determinar la significancia estadística de las variables latentes de primer orden, asumiendo la unidimensionalidad de los factores a un nivel del 5\% (Hair et al., 2004; Hoyle, 2015). Los indicadores de las tres dimensiones del CS definen adecuadamente estas dimensiones al presentar valores de correlación significativos (entre 0,591 y $0,806)$, el $P$ value de los errores se presenta significativo. El Alfa de Cronbach es significativo al presentar, para cada una de las tres dimensiones del CS, valores superiores a 0,856 . En lo que hace referencia a las dimensiones del $\mathrm{SCl}$, los indicadores de las tres dimensiones reflejan una confiabilidad en estas mediciones al presentar valores de correlación significativos (entre 0,560 y 0,748) con valores también significativos en los errores y valores del Alfa de Cronbach superiores a 0,878. Nuevamente, dos factores de la incertidumbre muestran un Alfa de Cronbach relativamente bajo $(<0,787)$ y dos de ellos son más adecuados $(>0,8)$.

\section{Análisis Factorial Exploratorio (EFA) y Confirmatorio (CFA)}

Dado que los cuestionarios no se han aplicado en el contexto colombiano y con el modelo propuesto, y conscientes que, según los resultados de análisis de fiabilidad y unidimensionalidad, algunos indicadores podrían no ser adecuados, los investigadores quisieron desarrollar la técnica de métodos multivariantes con el análisis factorial exploratorio (EFA) para identificar las dimensiones o factores subyacentes que permitan describir y comprender los datos con pocos factores (Hair et al., 2004). Como un mecanismo que valida el comportamiento de los datos obtenidos para este estudio, se dividió la muestra en dos segmentos independientes: la primera submuestra estuvo conformada por 60 encuestas con las cuales se generó el análisis factorial exploratorio (EFA) y para el análisis factorial confirmatorio (CFA) fueron utilizadas las restantes 172 encuestas. 
Los resultados del EFA identificaron que para los factores de SI, RC, SC y CC, los diferentes indicadores aportan de manera significativa y con mayor relevancia al respectivo factor definido, excepto un indicador del capital cognitivo, y algunos indicadores presentan aportes menos significativos a varios factores, Estos factores son medidos por lo menos con tres indicadores (Byrne, 2010; Hoyle, 2015). Sin embargo, los factores de II y Cl nuevamente no mostraron que sus indicadores estén direccionados a un único factor, especialmente el factor de integración con clientes. Por esta razón se evidenció que algunos indicadores de estos dos factores presentaban variada multidimensionalidad o no tenían significancia para algunos factores. Finalmente, se identificó para la incertidumbre un sólo factor con indicadores significativos. De acuerdo con los resultados evidenciados por el análisis unidimensional, el Alfa de Cronbach y el EFA, se decide suprimir varios indicadores por las inconsistencias antes definidas. En este sentido, se depuraron los indicadores P18 y P43 porque no eran consistentes teóricamente con el factor medido, y otros indicadores con significancia en varios factores. Igualmente se suprime la variable mediadora de la incertidumbre del ambiente (UE) porque no se evidencia consistencia en sus indicadores y se determina que con un sólo factor no se mide adecuadamente el constructo. Por estas razones, se suprimieron algunos indicadores, quedando las seis variables latentes de primer orden con el siguiente número de indicadores: RC 5, SC 5, CC 3, II 5, SI 9, y CI 5. El CFA, como técnica estadística, permite identificar patrones o interrelaciones entre varios factores latentes que son medidos mediante un grupo de indicadores observados, propuestos para cada variable. Como el CFA no especifica las relaciones direccionales, solamente muestra si los factores latentes están relacionados unos con otros (Hair et al., 2004), este estudio evaluó el CFA consolidado de los factores latentes y el de cada constructo del modelo teórico de primero y segundo orden. El objetivo del CFA fue, en primer lugar, verificar la estructura general del modelo para determinar si son identificados los seis factores latentes de primer orden resultantes y, en forma adicional, evaluar cada factor del modelo propuesto independientemente, para definir, así, si son necesarios los ajustes significativos.

El CFA del modelo completo identificó, efectivamente, los seis factores con sus respectivos indicadores, lo cual evidencia que los tres factores del capital social (CS) y los tres factores de la SCl son medidos adecuadamente por al menos tres indicadores. Para cada constructo, el primer modelo de CFA estuvo conformado por el constructo con una evaluación de primer orden (correlaciones) y de segundo orden (ponderaciones direccionales). Los índices usualmente más reconocidos para identificar el ajuste entre la matriz de covarianzas muestral y la matriz de covarianza poblacional del modelo propuesto, y que empleó esta investigación son los siguientes. El chi cuadrado o CMIN (minimum discrepancy, por sus siglas en ingles) define el grado de verosimilitud estadística y mide el nivel de ajuste de la prueba de la hipótesis del modelo (Hoyle, 2015). El CMIN/DF es un indicador del chi-cuadrado ajustado al dividirlo entre los grados de libertad del modelo; se sugiere que este sea menor que 2 (Byrne, 2010). El índice de bondad de ajuste del modelo GFI (Goodness-of-fit index, por sus siglas en inglés) trata de explicar el nivel de ajuste de varianza y covarianza de las matrices, independiente del tamaño muestral (Hoyle, 2015), y el CFI (Comparative fit index, por sus siglas en inglés) determina que la hipótesis del modelo defina adecuadamente los datos de la muestra, estos dos indicadores tienen un nivel recomendado mínimo de 0,90 (Hoyle, 2015). Finalmente, el RMSEA (Root mean square error of approximation, por sus siglas en inglés) trata de explicar qué tan bien podría el modelo ajustarse a la matriz de covarianza poblacional, si estuviera disponible, indicando la correlación residual media, que debe ser menor de 0,080 para ser aceptada y menor de 0,050 para identificar un muy buen ajuste (Hoyle, 2015). En la tabla 2 se muestran los índices de ajuste de bondad de los constructos SC y SCl, adicionalmente a los coeficientes estandarizados de las variables latentes del modelo de primer y segundo orden, desde el primer modelo al modelo ajustado de CFA. Se observa que se obtiene un ajuste adecuado para los dos constructos del modelo en el CFA, con índices de bondad de ajuste aceptables, luego de identificar modificaciones, al establecer covarianzas entre algunos indicadores de un mismo factor y suprimir algunos indicadores que permiten mejorar el ajuste del modelo.

Tabla 2: Índices de ajuste de bondad y coeficientes estandarizados del CFA para constructos CS y SCI

\begin{tabular}{|l|l|l|l|l|l|l|l|l|}
\hline \multicolumn{7}{|c|}{ CFA CS (Primer orden) } \\
\hline & CMIN & CMIN/DF & GFI & CFI & RMSEA & RC $\leftrightarrow$ CC & CC $\leftrightarrow$ SC & RC $\leftrightarrow$ SC \\
\hline Inicial & 176,859 & 2,807 & 0,865 & 0,922 & 0,103 & 0,571 & 0,763 & 0,521 \\
\hline Final & 90,175 & 1,840 & 0,919 & 0,969 & 0,070 & 0,576 & 0,768 & 0,543 \\
\hline CFA CS (Segundo orden con bootstrapping) \\
\hline
\end{tabular}




\section{Modelos de Ecuaciones Estructurales}

El modelo de ecuaciones estructurales (SEM), entendido como un conjunto de técnicas de análisis multivariante para estimación de relaciones de dependencias múltiples y cruzadas, permite identificar conceptos no observados en las relaciones y considerar el error de medida en la estimación (Hair et al, 2004).

En esta fase se busca una explicación de las relaciones entre los constructos (regresiones latentes). Las premisas de los datos para los modelos SEM es que sean continuos y normalmente distribuidos. Dado que esta investigación ha trabajado con cuestionarios con escala Likert de siete categorías, los estudios han demostrado que los métodos continuos pueden utilizarse con poca prevención cuando se tienen cuatro o más categorías (Byrne, 2010). La normalidad de los datos exige la distribución normal univariante de las diferentes variables y de la misma forma la normalidad multivariante. Las pruebas realizadas con la muestra obtenida identifican que los datos tienen normalidad univariante pero no así la normalidad multivariante. Una alternativa para trabajar con estos datos es el uso del procedimiento del bootstrapping, el cual consiste en considerar la muestra como si fuera la población y a partir de ésta construir aleatoriamente submuestras aleatorias con reemplazos (Byrne, 2010), con lo cual se probará, para este estudio, la estabilidad de los parámetros y de los índices de bondad de ajuste de los modelos propuestos.

\section{Modelo 1 - Impacto del RC en la SCI}

De acuerdo con la figura 2, se puede observar cómo el RC impacta en la SCl. Las cinco iteraciones del modelo son presentadas en la tabla 3, reflejando los cambios en los índices de ajustes del modelo. Dado que los índices obtenidos son aceptables y significativos, se sustenta la hipótesis $\mathrm{H} 2$ en el sentido que existe una relación directa, positiva y significativa del $\mathrm{RC}$ con el $\mathrm{SCl}$ en las empresas objeto del estudio.

Tabla 3: Índices de ajuste de bondad y coeficientes estandarizados del impacto del RC en la SCI

\begin{tabular}{|l|l|l|l|l|l|l|l|}
\hline ITERACIÓN & CMIN & CMIN/DF & $P$ & GFI & CFI & $R M S E A$ & $R C \rightarrow S C I$ \\
\hline Inicial & 201,469 & 1,752 & 0,000 & 0,872 & 0,936 & 0,066 & 0,598 \\
\hline Eliminar P17 & 170,280 & 1,703 & 0,000 & 0,885 & 0,943 & 0,064 & 0,428 \\
\hline Correl P27-P36 & 164,842 & 1,665 & 0,000 & 0,886 & 0,946 & 0,062 & 0,433 \\
\hline Correl P36-P37 & 156,313 & 1,595 & 0,000 & 0,894 & 0,952 & 0,059 & 0,453 \\
\hline Correl. P33-P36 & 148,190 & 1,528 & 0,001 & 0,902 & 0,958 & 0,056 & 0,466 \\
\hline Bootstrapping & 148,190 & 1,528 & 0,001 & 0,902 & 0,958 & 0,056 & 0,466 \\
\hline
\end{tabular}

Modelo 2 - Impacto del CC en la SCI

Tabla 4: Índices de ajuste de bondad y coeficientes estandarizados del impacto del CC en la SCI

\begin{tabular}{|l|l|l|l|l|l|l|l|}
\hline ITERACIÓN & CMIN & CMIN/DF & $P$ & GFI & CFI & RMSEA & $C C \longrightarrow$ SCI \\
\hline Inicial & 184,492 & 1,855 & 0,000 & 0,887 & 0,938 & 0,071 & 0,656 \\
\hline Eliminar P52 & 143,837 & 1,673 & 0,000 & 0,904 & 0,954 & 0,063 & 0,655 \\
\hline Correl P36-P37 & 137,655 & 1,619 & 0,000 & 0,911 & 0,958 & 0,060 & 0,661 \\
\hline Bootstrapping & 137,655 & 1,619 & 0,000 & 0,911 & 0,958 & 0,060 & 0,661 \\
\hline
\end{tabular}

De acuerdo con la figura 2, la variable latente endógena $\mathrm{SCl}(\mathrm{II}, \mathrm{Cl}, \mathrm{SI})$ es afectada por el $\mathrm{CC}$, una de las dimensiones de la variable latente exógena CS. Conforme las iteraciones de ajuste realizadas al modelo, presentadas en la tabla 4, se observa que los índices de ajuste del modelo son aceptables y significativos, lo cual permite inferir que hay evidencia significativa para determinar que existe una relación directa, positiva y significativa del CC en la SCl en empresas asociadas al sector real en Colombia, lo cual sustenta la hipótesis H3. 


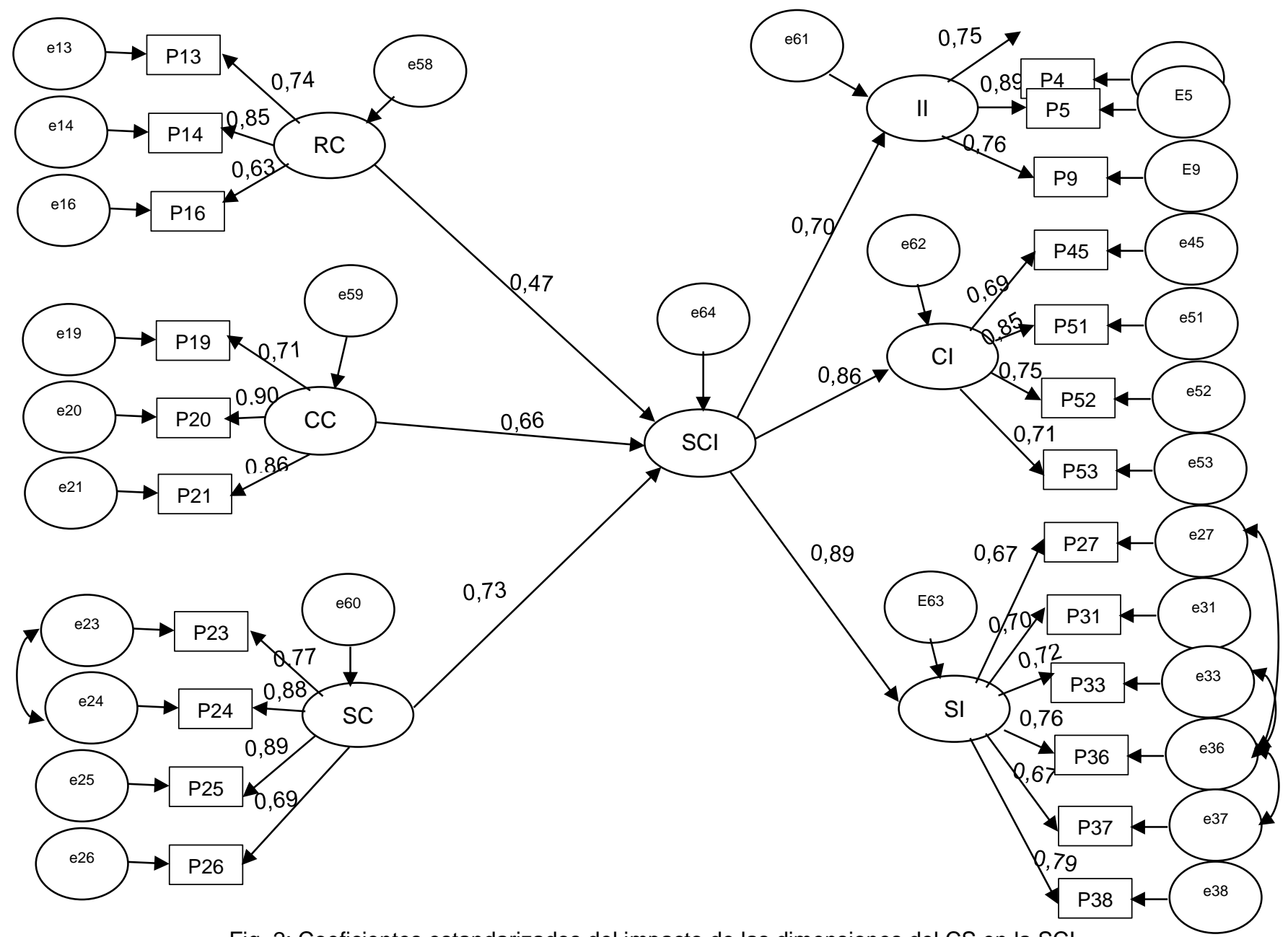

Fig. 2: Coeficientes estandarizados del impacto de las dimensiones del CS en la SCI

\section{Modelo 3-Impacto del SC en la SCI}

En la figura 2 se observa también que la variable latente endógena $\mathrm{SCl}$ es afectada por la SC. De acuerdo con los resultados de las iteraciones de ajuste, presentadas en la tabla 5, se identifica que hay evidencia significativa para determinar que existe una relación directa, positiva y significativa del SC en la SCI en las empresas asociadas al sector real en Colombia, lo cual sustenta la hipótesis H4.

Tabla 5: Índices de ajuste de bondad y coeficientes estandarizados del impacto del SC en la SCI

\begin{tabular}{|l|l|l|l|l|l|l|l|}
\hline ITERACIÓN & CMIN & CMIN/DF & P & GFI & CFI & RMSEA & SC $\rightarrow$ SCI \\
\hline Inicial & 230,490 & 1,759 & 0,000 & 0,874 & 0,942 & 0,067 & 0,745 \\
\hline Eliminar P33 & 198,437 & 1,726 & 0,000 & 0,885 & 0,948 & 0,065 & 0,742 \\
\hline Eliminar P22 & 171,279 & 1,713 & 0,000 & 0,894 & 0,950 & 0,065 & 0,732 \\
\hline Correl. P35-P36 & 161,212 & 1,628 & 0,000 & 0,898 & 0,956 & 0,061 & 0,733 \\
\hline Bootstrapping & 161,212 & 1,628 & 0,000 & 0,898 & 0,956 & 0,061 & 0,733 \\
\hline
\end{tabular}

Modelo 4 - Impacto del CS en la SCI

En la figura 3 se presenta el modelo SEM, de segundo orden, donde se identifican las relaciones y factores de carga significativos entre la variable latente endógena $\mathrm{SCI}(\mathrm{II}, \mathrm{CI}, \mathrm{SI}$ ) afectada por la variable latente exógena CS (RC, CC y SC). Conforme a las iteraciones de ajuste efectuadas al modelo, presentadas en la tabla 6 , hay evidencia significativa para determinar que existe una relación directa, positiva y significativa del CS en la SCl en empresas asociadas al sector real en Colombia, lo cual sustenta la hipótesis H1. 


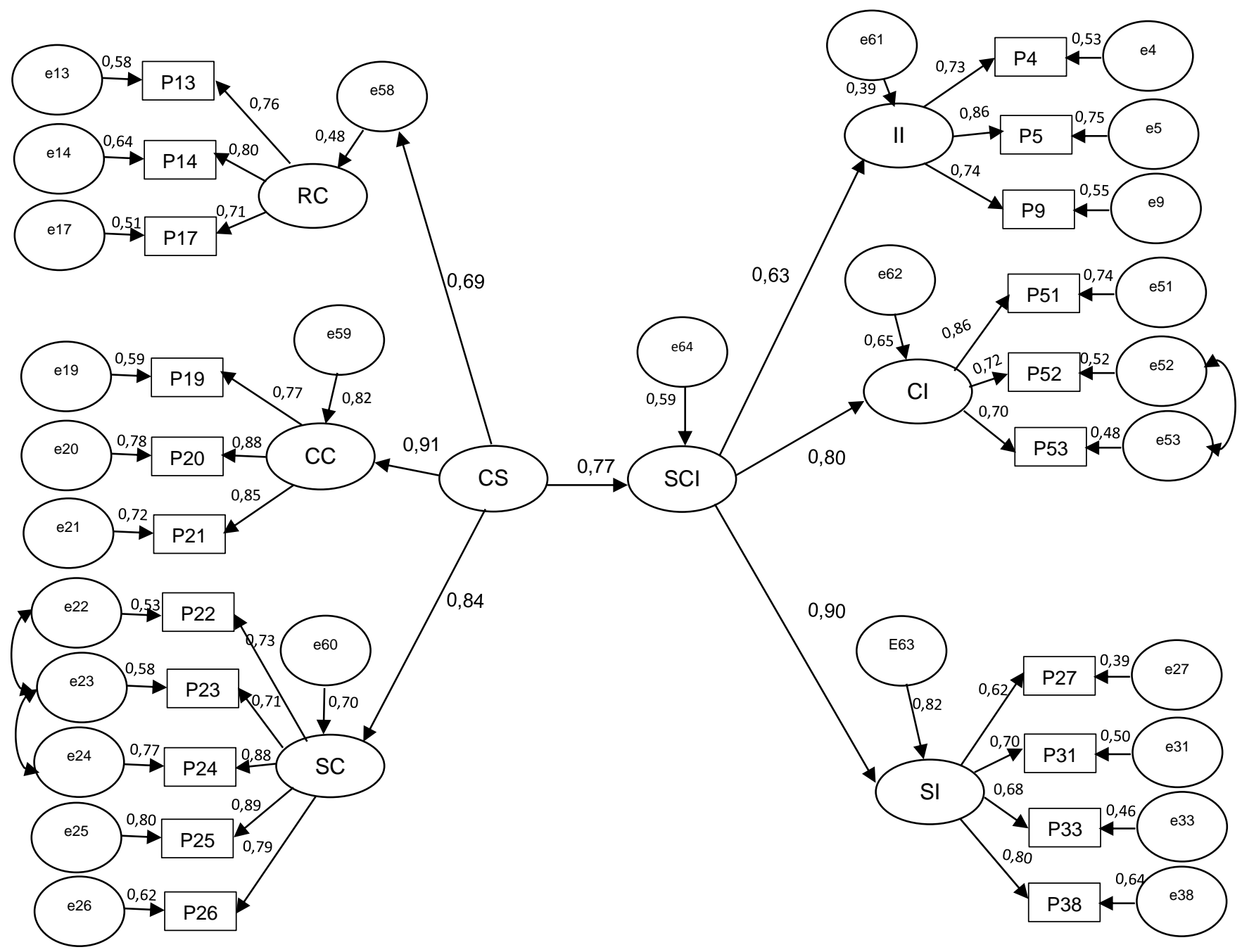

Fig. 3: Coeficientes estandarizados del impacto del CS en la SCI

Tabla 6: Índices de ajuste de bondad y coeficientes estandarizados del impacto del CS en la SCI

\begin{tabular}{|l|l|l|l|l|l|l|l|}
\hline Iteración & CMIN & CMIN/DF & $P$ & GFI & CFI & RMSEA & CS $\rightarrow$ SCI \\
\hline Inicial & 434,504 & 1,627 & 0,000 & 0,833 & 0,933 & 0,061 & 0,723 \\
\hline Eliminar P36 & 383,643 & 1,572 & 0,000 & 0,846 & 0,940 & 0,058 & 0,744 \\
\hline Eliminar P37 & 325,338 & 1,465 & 0,000 & 0,861 & 0,953 & 0,052 & 0,762 \\
\hline Correl. P52-P53 & 319,862 & 1,447 & 0,000 & 0,862 & 0,956 & 0,051 & 0,763 \\
\hline Eliminar P16 & 277,176 & 1,379 & 0,000 & 0,874 & 0,964 & 0,047 & 0,769 \\
\hline Eliminar P45 & 250,378 & 1,383 & 0,000 & 0,880 & 0,965 & 0,047 & 0,769 \\
\hline Bootstrapping & 250,378 & 1,383 & 0,000 & 0,880 & 0,965 & 0,047 & 0,769 \\
\hline
\end{tabular}

Modelo 5 - El tamaño (S) y la incertidumbre ambiental (UE) I

En este modelo se busca identificar el tamaño (S) de las organizaciones como una variable moderadora de la relación entre la variable latente exógena CS y la variable latente endógena SCI.

Tabla 7: Índices de ajuste de bondad y coeficientes estandarizados del tamaño como mediador entre el CS y la SCI

\begin{tabular}{|l|l|l|l|l|l|l|l|l|l|}
\hline Iteración & CMIN & CMIN/DF & $P$ & GFI & CFI & RMSEA & CS $\rightarrow S C I$ & $C S \rightarrow S$ & $S \rightarrow S C l$ \\
\hline Inicial & 389,267 & 1,937 & 0,000 & 0,827 & 0,908 & 0,074 & 0,330 & 0,279 & 0,598 \\
\hline Bootstrapping & 389,267 & 1,937 & 0,000 & 0,827 & 0,908 & 0,074 & 0,33 & 0,279 & 0,598 \\
\hline
\end{tabular}


Los resultados, presentados en la tabla 7, permiten evidenciar que el tamaño modera de forma medianamente directa la relación. Los indicadores de ajuste del modelo son apenas aceptables. Esto permite evidenciar que no existe una moderación inversa del S en la relación del CS y el SCI en el sector real en Colombia, por lo cual se rechaza la hipótesis H5. De igual forma, como la variable de incertidumbre ambiental (UE) no obtuvo indicadores aceptables, no se pudo corroborar la hipótesis $\mathrm{H} 6$.

\section{DISCUSIÓN}

Los resultados obtenidos por este estudio permiten aportar al conocimiento sobre la SCl así como a su relación con factores blandos de relacionamiento consolidados en el concepto de CS. Es importante señalar que, por ser un modelo de segundo orden, adquieren especial importancia los indicadores observables que miden las variables latentes de primer orden, que para este caso fueron evaluados a través del análisis de fiabilidad del Alfa de Cronbach, el cual los identificó como significativos. Adicionalmente, el análisis de unidimensionalidad permitió corroborar que las variables observables están midiendo cada factor asociado. Los factores de ponderación de las variables latentes de segundo orden sobre las variables latentes de primer orden también identifican relaciones significativas.

Estas pruebas, junto con el EFA y el CFA, permitieron filtrar algunos indicadores porque no medían adecuadamente los factores asociados, así se pudo identificar que los factores RC, CC y SC miden adecuadamente al constructo CS, en consonancia con la propuesta de Nahapiet y Ghoshal (1998). De otra parte, la SCl es medida adecuadamente por la SI (Flynn et al., 2010), pero no es tan clara en la II y la CI, en las cuales algunos indicadores no están aportando significativamente al factor asociado. Esta misma inconsistencia se presenta para las dimensiones del UE, por lo cual esta variable no pudo ser corroborada (Boon-itt y Wrong, 2010) y considerada. El análisis confirmatorio evidencia que sin los indicadores suprimidos desde los análisis previos, se logra definir adecuadamente las tres dimensiones del CS y las tres de la SCI, con al menos tres indicadores y con un peso significativo mayor que 0,4 (Byrne, 2010).

El estudio realizado permite evidenciar una relación directa positiva y significativa entre las tres dimensiones del CS y la SCI. La dimensión del SC, seguida por el CC, ponderan significativamente en la SCI y, en menor proporción, el RC, con índices aceptables de ajuste de los modelos. Esto evidencia que, para los industriales del sector real, en Colombia, el establecer vínculos con diferentes empresas en su segmento es primordial para los procesos de integración y que su prioridad es identificar que estas relaciones estén basadas en un lenguaje y cultura comunes, para definir propósitos en conjunto dentro de la SCM, lo cual es consistente con los estudios de AISagheer et al. (2011) y, Lambert y Cooper (2000). Con menor relevancia, se identifica el RC a través del establecimiento de lazos de confianza con sus aliados. Estas tres dimensiones son positivas y significativas, lo cual sustenta los estudios de Autry y Griffis (2008) que identifican una relación positiva del capital estructural y relacional con la gestión de la innovación en la SCM, quienes encuentran una relación significativa entre el capital estructural y relacional con la integración.

De acuerdo con los resultados, se evidencia una relación directa, positiva y significativa entre el CS y la SCI, con índices de bondad de ajuste del modelo muy adecuados, lo cual permite establecer que los elementos blandos y, específicamente, el capital social juega un rol preponderante, al ser éste identificado como un factor clave para el desarrollo de las funciones organizacionales y para fortalecer la integración interorganizacional, como lo mencionan Son et al. (2016). También es resaltable que el CS, como constructo, tiene un mayor efecto en la $\mathrm{SCl}$ que cada una de sus dimensiones independientemente consideradas, y que pondera en forma significativa en cada una de estas dimensiones, en especial el CC y el SC, lo cual evidencia la importancia de trabajar en todas estas dimensiones para lograr un efecto destacado en los procesos de $\mathrm{SCl}$ mediante la construcción de una red de relaciones que permita proporcionar recursos a las organizaciones y adquirir conocimiento de otros miembros de la red (Adler y Kwon, 2002).

De otra parte, la investigación evidencia que la relación entre el CS y la SCI no es, necesariamente, mediada por el tamaño, y esta podría ser una relación directa y no inversa. La medición de la incertidumbre del entorno no fue la adecuada y es necesario identificar mediciones más adecuadas. Esto podría evidenciar que el concepto de CS, independientemente de otras variables, es un factor relevante y significativo para determinar si las organizaciones están en posibilidad de integrar sus procesos con otras organizaciones en sus cadenas y redes de abastecimiento. En consonancia con lo anterior, se evidencia, a partir del estudio, que el CS constituye un concepto integral y multidimensional que explica más significativamente las relaciones con la $\mathrm{SCl}$ que otros elementos blandos de relacionamiento. Esto podría ser interpretado como el hecho que, cuando se desea establecer la $\mathrm{SCl}$, las empresas no solamente tienen que estructurar una importante red de relaciones con sus aliados estratégicos de la cadena sino, también, compartir una cultura organizacional similar con metas comunes y, finalmente, generar compromiso y confianza entre las partes, lo cual, dada la relevancia de la SI y $\mathrm{Cl}$ en el modelo, es consistente con varios estudios que proponen una relación positiva entre el CS y las relaciones con proveedores y clientes en la SCM (Son et al., 2016; Villena et al., 2011). 


\section{COMENTARIO FINAL}

De acuerdo con los resultados de esta investigación, se puede evidenciar que existe una relación significativa entre el CS y la SCl en las empresas asociadas al sector real en Colombia. Esto puede definirse como la necesidad que tienen las empresas que quieran, mediante la integración de sus procesos de negocio con otras organizaciones de su SCM, garantizar los recursos requeridos en el largo plazo, el trabajar en los siguientes tres aspectos: primero, construir una sólida red de relaciones a diferentes niveles con distintas empresas en esta red; posteriormente, identificar entre estos aliados aquellos con los cuales se comparte una cultura y propósitos comunes, para estrechar estos vínculos, y, finalmente, establecer con ellos relaciones centradas en la confianza y el compromiso hacia proyectos futuros.

El capital social representa un factor significativo como determinante directo de la SCl. El tamaño, como variable mediadora, no se identifica en esta investigación como un determinante inverso en la relación, lo cual implica que el tamaño de las organizaciones no facilita o impide estos procesos de integración. La UE requiere identificar nuevos indicadores que la permitan medir adecuadamente. De otra parte, la investigación sugiere que el mayor impacto que se puede lograr en los proyectos de la SCl se obtendría cuando se trabaja en las tres dimensiones del CS como conjunto, lo cual permite afirmar que una empresa obtendría mayores beneficios en la integración de procesos de negocios con sus aliados de la SCM si desarrolla las tres dimensiones del CS, consistentemente, en una visión de largo plazo. De otra parte, se puede evidenciar la robustez de la metodología SEM, la cual permitió generar confiabilidad en los análisis.

De esta manera, la presente investigación proporciona un aporte importante en la extensión del conocimiento de la SCI y la identificación y manejo de las dimensiones del CS para mejorar la competitividad organizacional. Adicionalmente, a diferencia de diversos estudios que evalúan las relaciones diádicas, esta investigación buscó identificar las relaciones de la SCM en un entorno de red que permiten identificar altos niveles de integración. Por último, este estudio ha verificado que el capital social es un adecuado determinante de la SCl dado que evalúa las relaciones entre diferentes actores de la cadena de abastecimiento para permitirles el acceso a los recursos que requieren, $y$, por lo tanto, un aumento en el CS contribuye a todas las funciones organizacionales, entre ellas a la integración.

\section{CONCLUSIONES}

De acuerdo al trabajo presentado y a los resultados obtenidos, se pueden plantear las siguientes conclusiones principales:

1.- Las tres dimensiones del CS referentes al RC, CC y SC consideradas independientemente, reflejan un relación positiva y significativa con la $\mathrm{SCl}$ en las empresas asociadas al sector real en Colombia.

2.- EI CS es un determinante directo y significativo de la $\mathrm{SCl}$ en las empresas asociadas al sector real en Colombia, y más relevante que cada una de sus dimensiones.

3.- El tamaño de las organizaciones no se identifica como una variable medidora inversa entre el CS y la SCI en las empresas asociadas al sector real en Colombia

\section{REFERENCIAS}

Adler, P. S. y S.W. Kwon, Social capital: Prospect for a new concept, Academy of Management Review, 27, 17-40 (2002)

Aldana-Bernal, J.C. y C.A. Bernal-Torres, Factores Blandos en la Gestión de Integración de las Cadenas y/o Redes de Abastecimiento: Aproximación a un Modelo Conceptual, doi.org/10.4067/S0718-07642018000200103, Información Tecnológica, 29(2), 103-114 (2018)

AlSagheer, A., H.B. Mohammed y otros tres autores, Impact of Supply Chain Integration on Business Performance and its Challenges, International Business \& Economics Research Journal, 10(12), 79-93 (2011)

Autry, C.W. y S.E. Griffis, Supply Chain Capital: The Impact of Structural and Relational, Linkages on Firm Execution and Innovation, Journal of Business Logistics, 29(I), 157-173 (2008)

Bastas, A. y K. Liyanage, Sustainable Supply Chain Quality Management: A systematic review, Journal of Cleaner Production, 181, 726e744 (2018)

Boon-itt, S. y C. Wong, The Moderating Effects of Technological and Demand Uncertainties on the Relationship between Supply Chain Integration and Customer Delivery Performance, International Journal of Physical Distribution \& Logistics Management, 41 (3), 253-276 (2010)

Borgatti, S.P. y X. Li, On Social Network Analysis in a Supply Chain Context, Journal of Supply Chain Management, 45(2), 5-22 (2009) 
Byrne, B.M., Structural Equation Modelling with Amos: Basic Concepts, Applications, and Programming, $2^{\text {nd }}$ Ed., 35-352, Routledge, New York (2010)

Flynn, B.B., B. Huo y X. Zhao, The Impact of Supply Chain Integration on Performance: a Contingency and Configurational Approach, Journal of Operations Management, 28(1), 58-71 (2010)

Giménez, C., T. Van der Vaart y D.P. Van Donk, Supply Chain Integration and Performance: the Moderating Effect of Supply Complexity, International Journal of Operations \& Production Management, 32(5), 583-610 (2012)

Hair, J.F., R.E. Anderson y otros dos autores, Multivariante, $5^{\text {th }}$ Ed., New York, Prentice Hall (2004)

Horn, P., P. Sheffler y H. Schiele, Internal Integration as a Pre-condition for External Integration in Global Sourcing: A Social Capital Perspective, Int. Journal Production Economics, 153, 54-65 (2014)

Hoyle, R.H., Handbook of Structural Equation Modeling, 43-144, The Guilford Press, New York (2015)

Inkpen, A.C. y E.W.K. Tsang, Social Capital, Networks, and Knowledge Transfer, Academy of Management Review, 30(1), 146-165 (2005)

Katunzi, T.M., Obstacles to Process Integration along the Supply Chain: Manufacturing Firms Perspective, International Journal of Business and Management, 6(5), 105-113 (2011)

Lambert D.M. y M.C. Cooper, Issues in Supply Chain Management, Industrial, Marketing Management, 29, 65-83 (2000)

Lockamy, A. y K. McCormack, The Development of a Supply Chain Management Process Maturity Model using the Concepts of Business Process Orientation, Supply Chain Management: An International Journal, 9(4), 272-8 (2004)

Matthews, R.L. y P.E. Marze, Social Capital, a Theory for Operations Management: a Systematic Review of the Evidence, International Journal of Production Research, 50(24), 7081-7099 (2011)

Mentzer, J.T., W. DeWitt y otros cinco autores, Defining Supply Chain Management, Journal of Business Logistics, 22(2), 1-25 (2001)

MinCIT, Ministerio de Comercio Industria y Turismo Oficina de Estudios Económicos, Publicaciones (2017)

Morash, E.A. y S.R. Clinton, Supply Chain Integration: Customer Calue Through Collaborative Closeness versus Operational Excellence, Journal of Marketing Theory and Practice, 6(4), 104-21 (1998)

Nahapiet, J. y S. Goshal, Social Capital Intellectual Capital and the Organizational Advantage, Academy of Managemeni Review, 23 (2), 242-266 (1998)

Naslund, D. y H. Hulthen, Supply Chain Management Integration: A Critical Analysis, Benchmarking: An International Journal, 19 (4/5), 481-501 (2012)

Roden, S. y B. Lawson, Developing Social Capital in Buyer-Supplier Relationships: The Contingent Effect of Relationshipspecific Adaptations, Int. Journal. Production Economics, 151, 89-99 (2014)

Roldán, S., Contexto de la Problemática de la Cadena de Abastecimiento desde la Perspectiva Latinoamericana: Una Mirada Hacia el Enfoque de Direccionamiento de las Empresas en el Valle Del Cauca, Global Conference on Business and Finance Proceedings, 10(1), 1313-1323 (2015)

Ruiz, A., F. Mahmoodi y J. Ayala, Supply Chain Management Research in Latin America: a Review, Supply Chain Forum and International Journal, 13(1), 20-36 (2011)

Simanca, M., L. Montoya y C. Bernal, Gestión del Conocimiento en la Cadena Láctea Colombia, Revista Información Tecnológica, 27(3), 93-106 (2016)

Son, B.G., C. Kocabasoglu-Hillmer y S. Roden, A Dyadic Perspective on Retailer-Supplier Relationships Through the Lens of Social Capital, Int. J. Production Economics, 178, 120-131 (2016)

Torres, A. y J.G. García, Formas de Gobernación de la Cadena de Abastecimiento: Revisión Bibliográfica y Propuesta de Modelo de Investigación, Cuadernos de Administración, 21(35), 65-91 (2008)

Villena, V.H., E. Revilla y T.Y. Choi, The Dark Side of Buyer-Supplier Relationships: A Social Capital Perspective, doi: 10.1016/j.jom.2010.09.001, Journal of Operations Management, 29, 561-576 (2011) 
